\title{
Methods for optimizing routes in digital logistics
}

\author{
Lubov Ivanova ${ }^{1}$, Andrey Kurkin ${ }^{1}$, and Sergei Ivanov ${ }^{2, *}$ \\ ${ }^{1}$ Saint Petersburg State Marine Technical University, 3, Lotsmanskaya, St. Petersburg, Russia \\ ${ }^{2}$ ITMO University, 49, Kronverksky Pr., St. Petersburg, Russia
}

\begin{abstract}
The current problem of digital logistics is investigated - the calculation of optimal routes for freight transportation by computer means to reduce time and distance. Heuristic methods used in logistics for constructing optimal routes are considered. A comparative analysis of ten methods for solving the optimization problem of the "nondeterministic polynomial time" complexity class traveling salesman is carried out. The study performs a comparative analysis of the following methods: "convex hull, cheapest insertion and angle selection", "greedy", "greedy-cycle", "integer-linear-programming", "or-opt", "or-zweig", "remove crossings", "space filling curve", "simulated annealing", "two-opt". A computational experiment is performed, on the basis of which the accuracy and computational complexity of the considered methods are estimated. The results of the computational experiment show the construction of the optimal route by the "integer-linear-programming" method and the highest computation speed for the "greedy" method. Application of the "integerlinear-programming" method in logistics is the most accurate at the optimal time for calculating efficient routes of freight traffic.
\end{abstract}

\section{Introduction}

The study examines the urgent problem of logistics - determining the optimal routes for freight transportation to reduce the time of transportation. Many scientific works are devoted to the problems of logistics automation and intelligent transport systems [1-3].

Modern information technologies open up new possibilities for improving and developing logistics. With the help of such technologies, it is possible to determine much more accurate data on logistics processes and automatically develop rational management decisions.

The main criteria for optimizing freight traffic are delivery time and route distance. In digital logistics, computer tools are widely used for the automated calculation of optimal routes through mathematical optimization methods. Many heuristic methods and their implementation in computer programs are known to determine the optimal route.

Let's consider the main heuristic methods for solving the problem of determining the optimal route.

Heuristic methods, which are based on probabilistically directed enumeration and apply elements of randomness, were formed relatively recently and are dynamically developing. Heuristic algorithms include bioinspired algorithms: genetic, ant, swarm. Research into solving the traveling salesman problem is progressing towards expanding the input space.

*Corresponding author: 45is@mail.ru 
Increasing the speed of the computer increases the search space in solving the salesman problem. The efficiency of data processing algorithms and the development of software serves as the basis for the evolution of information technology.

The methods are applied to the solution of the combinatorial optimization problem of the NP class traveling salesman [4-6]. In the setting of the traveling salesman problem, it is necessary to determine the optimal route passing through the indicated nodes once, with a subsequent return to the original node. In the mathematical setting of the traveling salesman problem, it is required to determine the optimal closed route in the graph, visit all the vertices of the graph, and then return to the original vertex. For the top of the graph, a transport node is taken, which in its simplest form is represented by an intersection. Many scientific studies are devoted to mathematical methods of graph theory [7,8]. Provided that each vertex of the graph is included in the route, a Hamiltonian cycle is defined once. To simplify the task, the graph is considered fully connected. If there is no connection between the nodes, an edge with the maximum length is added. The Euclidean distance is used as a metric in the traveling salesman problem [9]. In the NP class problem of the traveling salesman, the complexity estimate is not polynomial. When the number of nodes is on the order of a hundred, the solution of the NP class problem by exhaustive search of routes will take infinite time to search. Therefore, to solve problems of the class NP, heuristic methods are used.

In the study, we will consider the application of heuristic methods: "convex hull, cheapest insertion and angle selection" [10], "greedy" [11], "greedy-cycle" [12], "integer-linearprogramming" [13], "or -opt "[14]," or-zweig "[15]," re-move crossings "[16]," space filling curve "[17]," simulated annealing "[18]," two-opt "[19 ]. However, not all methods provide an optimal route and acceptable computational complexity.

The purpose of this study was to carry out a comparative analysis of the accuracy and computational complexity of the considered methods.

The main goal of the article is to determine the optimal route optimization method in digital logistics in terms of accuracy and computation time based on a computational experiment.

\section{Materials and Methods}

One of the most well-known combinatorial optimization problems (the traveling salesman problem) is formulated as follows: a salesman leaving a city must visit ( $n-1)$ other cities and return to the starting point. The distances between all the given cities are known. It is required to establish the order of visiting each city so that the total distance traveled is minimal.

Despite the simplicity of the statement of the traveling salesman problem, the computational difficulty of the solution should be noted. One of the solutions to this problem is the method of full enumeration of all possible options. Since the choice of the traveling salesman at each step depends on the number of cities that he has not yet visited, we get there are $(\mathrm{n}-1)$ ! routes for the traveling game.

Therefore, the search space has an exponential relationship with the number of cities.

One of the methods of multidimensional mathematical optimization (differential evolution) uses some elements of genetic algorithms and belongs to the class of stochastic algorithms. The method is easy to implement and use, easy to parallelize.

It should be noted that differential evolution is a direct optimization method designed to find the global minimum (or maximum) of non-differentiable, non-linear, multi-extremal functions of many variables.

On small sets of variables, the differential evolution method is significantly inferior to the exhaustive search method [21]. To enumerate a small number of combinations, it is necessary to create large populations and perform a large amount of mutations over vectors, which is a significant drawback of the differential evolution method. 
Genetic algorithms are computational search algorithms mobilized by evolutionary processes [22]. Algorithms are used to solve optimization and modeling problems by methods of random selection, combination and variation of the required parameters using mechanisms similar to natural selection in nature. These algorithms are used to solve a wide range of problems, including logistics (for example, solving the problem of planning fuel consumption).

Ant Colony Optimization (ACO) is one of the most effective algorithms for finding approximate solutions to the traveling salesman problem, and solving similar problems of finding routes on graphs [23-24]. Artificial ants in ACO are stochastic methods of constructing potential solutions to a problem using adapted (artificial) information based on available heuristic data. For example, an algorithm for optimizing ant colonies was proposed to determine the method for allocating jobs, planning tools in order to minimize the duration of processing plans in the production system.

The solution of the traveling salesman problem by the united intelligence of a group of experts shows the effectiveness of the genetic consultation method, increases the thinking abilities of the group. The effectiveness of the method increases the accuracy of the choice of interaction rules and the correct paradigm for modifying the collective method for solving problems.

This method can be used to solve complex optimization problems in order to get effective results in a short time. When using the method of genetic consultation, it is necessary to take into account the intellectual potential existing in the organization.

The method "Convex hull, Cheapest insertion and Angle selection" [10] was developed in 1985 by Golden and Stewart on the basis of the representation of the convex hull as a stretched rubber band along the nodes. The method finds the place of insertion into the route of each point without touching the tape with a minimum increase in the route. A node is added to the route with the greatest angle until all nodes are included in the route

The "Greedy" method [11] performs the optimal local one at each step to determine the global optimum. It is known that the solution to the NP class traveling problem by the "Greedy" method may not be optimal. The Greedy method is the nearest neighbor algorithm. Bypass nodes are sequentially included in the route, so that the included node is the closest among all to the last selected one.

The "Greedy-Cycle" method [12] uses the construction of loops based on the "Greedy" methods. The constructed cycle is taken as the optimal route.

The methods of "Integer Linear Programming" [13] for solving the optimization problem with integer variables include the method of cutting planes and the method of branches and bounds. The section plane method solves a simplified linear programming problem with the addition of constraints on non-integer solutions. The branch-and-bound method for the traveling salesman problem was developed in 1963 by a group of scientists (J. Little, C. Murthy, D. Sweeney, C. Carol). The branch and cut method has polynomial complexity and is a combination of the branch and cut method with the cutting plane method.

The "OrOpt" method [14] was developed in 1976 and considers a complete graph and removes the edges of one, two or three nodes of the route and replaces them with edges that decrease the length of the route. If all the edges are replaced and the length does not decrease, then the optimal route is determined.

The OrZweig method [15] was developed in 1995 by the Zweig scientist based on a modification of the OrOpt method using Delaunay triangulation for neighboring nodes.

The "Remove Crossings" method [16] removes the intersected edge in a graph with a large length.

The "Space Filling Curve" method [17] uses a Peano curve whose image contains a hypercube to construct the optimal route. A space-filling curve is constructed as the limit of a sequence of piecewise-linear continuous curves. 
The "Simulated Annealing" method [18] for solving the discrete optimization problem is based on the imitation of the physical process of annealing of metals developed by $\mathrm{N}$. Metropolis. In the method, a point with a minimum of a numerical function is determined by sequential calculation of points in space.

The "TwoOpt" method [19] for a complete graph removes two edges in a route and replaces them with edges with a shorter length, decreasing the total length of the route at each step until all edges are considered. class.

The presented ten methods are the most used for solving optimization problems of the NP

\section{Results}

A computational experiment was carried out to calculate the optimal routes by the ten methods considered for from 10 to 75 nodes.

Figure 1 shows the dependence of the computational time on the number of nodes of the route built by the following methods: "convex hull, cheapest insertion and angle selection", "greedy", "greedy-cycle", "integer-linear-programming", "or-opt "," or-zweig "," remove crossings "," space filling curve "," simulated annealing "," two-opt". The x-axis is the number of route nodes, the y-axis is the computation time in seconds.

Figure 1 shows the fastest computation time for the "greedy", "space filling curve" methods and the longest time for the "simulated annealing" method.

Figure 2 shows transport routes for 75 nodes constructed by the methods a) "convex hull, cheapest insertion and angle selection", b) "greedy", c) "greedy-cycle", d) "integer-linearprogramming", e ) "or-opt", f) "or-zweig", g) "remove crossings", h) "space filling curve", j) "simulated annealing", k) "two-opt".

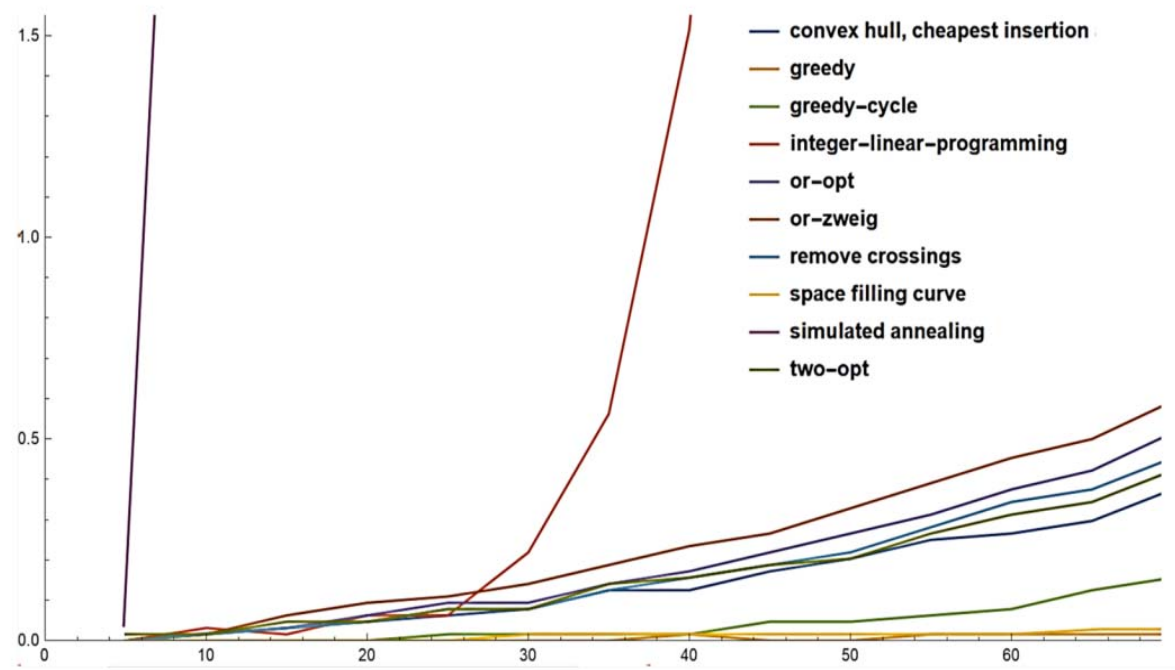

Fig. 1. Dependences of the computational time on the number of route nodes. 
a)"convex hull, cheapest insertion and angle selection"

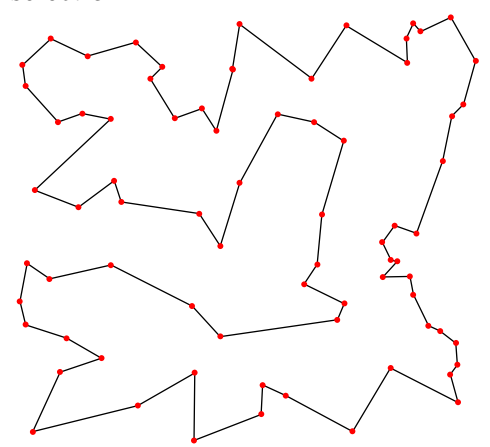

b)"greedy"

c)"greedy-cycle"

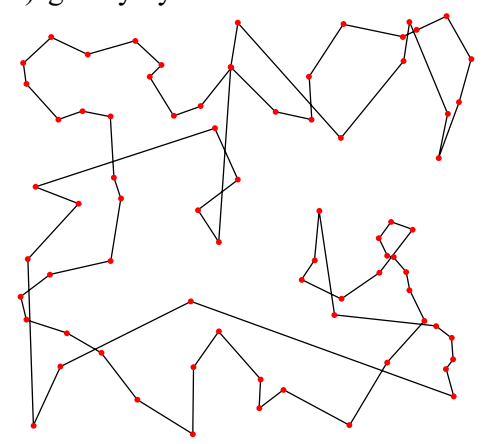

e)"or-opt"

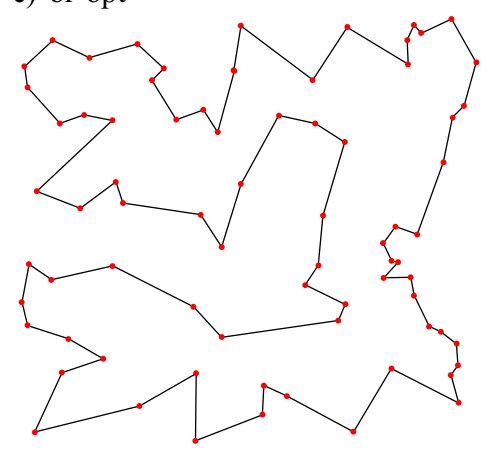

g)"remove crossings"

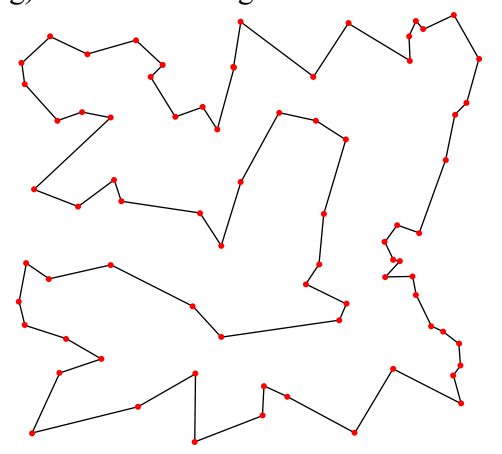

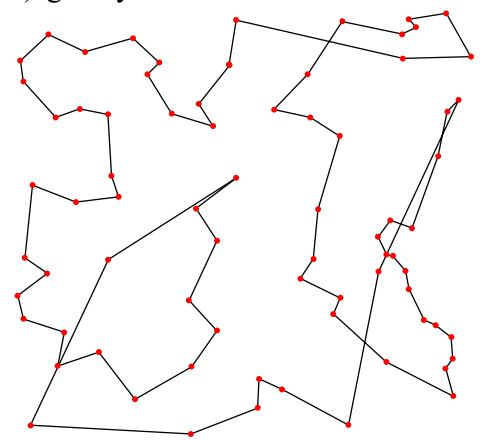

d)"integer-linear-programming"

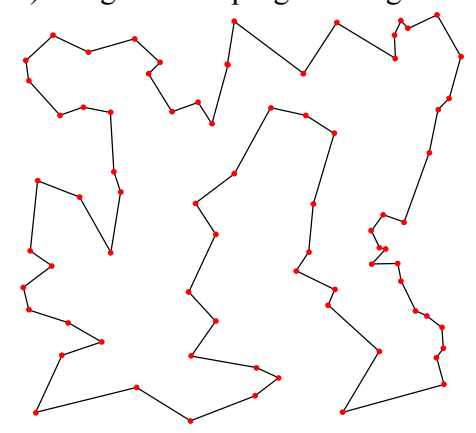

f)"or-zweig",

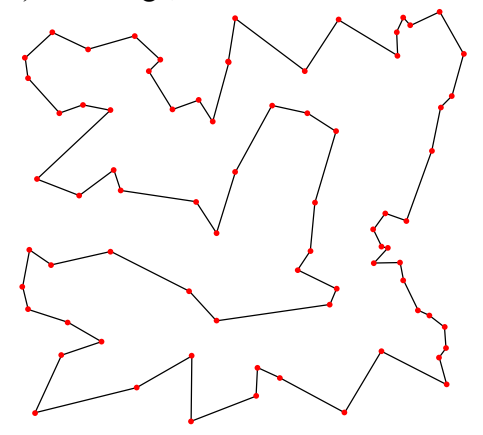

h)"space filling curve"

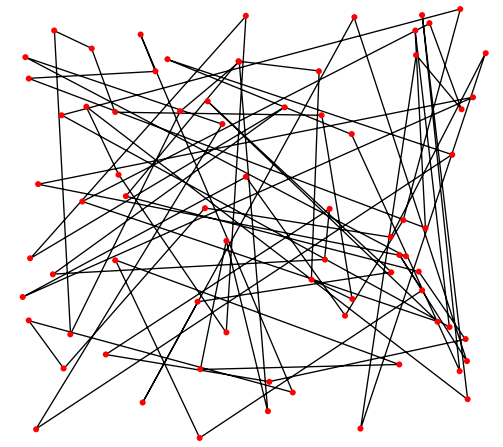


j)"simulated annealing"

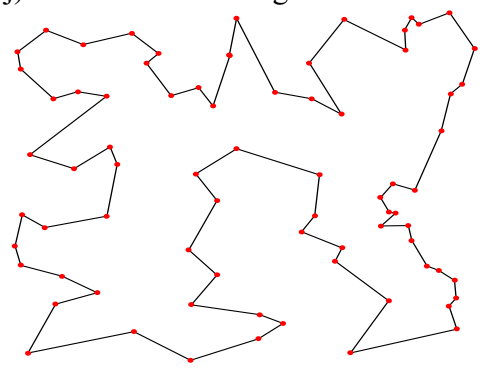

k)"two-opt"

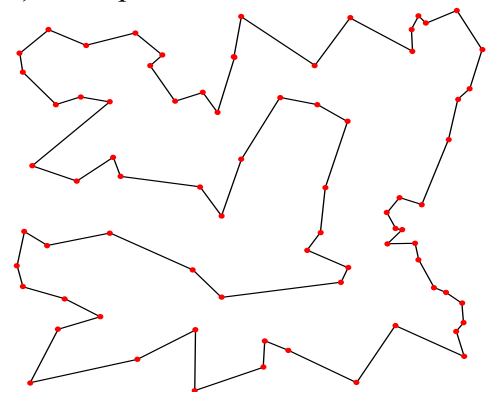

Fig. 2. Transport routes for 75 knots.

Figure 2 shows the optimal route with the shortest length, constructed using the "integerlinear-programming" method. The presented figures were created by the authors using a specialized mathematical software package.

Table 1 shows the results of a computational experiment - the length of the route and the computation time by ten methods for 75 nodes.

Table 1. Computational Experiment Results.

\begin{tabular}{|l|c|c|}
\hline \multicolumn{1}{|c|}{ Method } & Route length (km) & $\begin{array}{c}\text { Computation time } \\
\text { (s) }\end{array}$ \\
\hline $\begin{array}{l}\text { "convex hull, cheapest insertion } \\
\text { and angle selection" }\end{array}$ & 727.579 & 0.375 \\
\hline "greedy" & 847.938 & 0.016 \\
\hline "greedy-cycle" & 979.456 & 0.172 \\
\hline "integer-linear-programming" & 703.577 & 13.625 \\
\hline "or-opt" & 727.579 & 0.516 \\
\hline "or-zweig" & 727.579 & 0.578 \\
\hline "remove crossings" & 727.579 & 0.453 \\
\hline "space filling curve" & 4041.44 & 0.017 \\
\hline "simulated annealing" & 711.008 & 51.563 \\
\hline "two-opt" & 727.579 & 0.469 \\
\hline
\end{tabular}

Comparative analysis of the data in the table shows the best "integer-linear-programming" method for building the shortest route: and the fastest computation speed for the "greedy" method.

The obtained results of the computational experiment agree with the asymptotic estimates of the computational complexity of the methods.

\section{Discussion}

As a result of the experiment, a comparative analysis of the methods: "convex hull, cheapest insertion and angle selection", "greedy", "greedy-cycle", "integer-linear-programming", "oropt", "or-zweig", "remove crossings", "space filling curve", "simulated annealing", "twoopt".

The calculation time and route length were determined as criteria for optimization.

The results of the computational experiment show the construction of the optimal route by the "integer-linear-programming" method and the highest computation speed for the "greedy" method. The application of the "integer-linear-programming" method in logistics is the most accurate at the optimal time to calculate efficient routes for freight traffic. The results obtained allow us to choose the best methods for digital logistics. 
As an application, the optimal transport route was determined using the "integer-linearprogramming" method for 75 nodes (Figure 3 ).

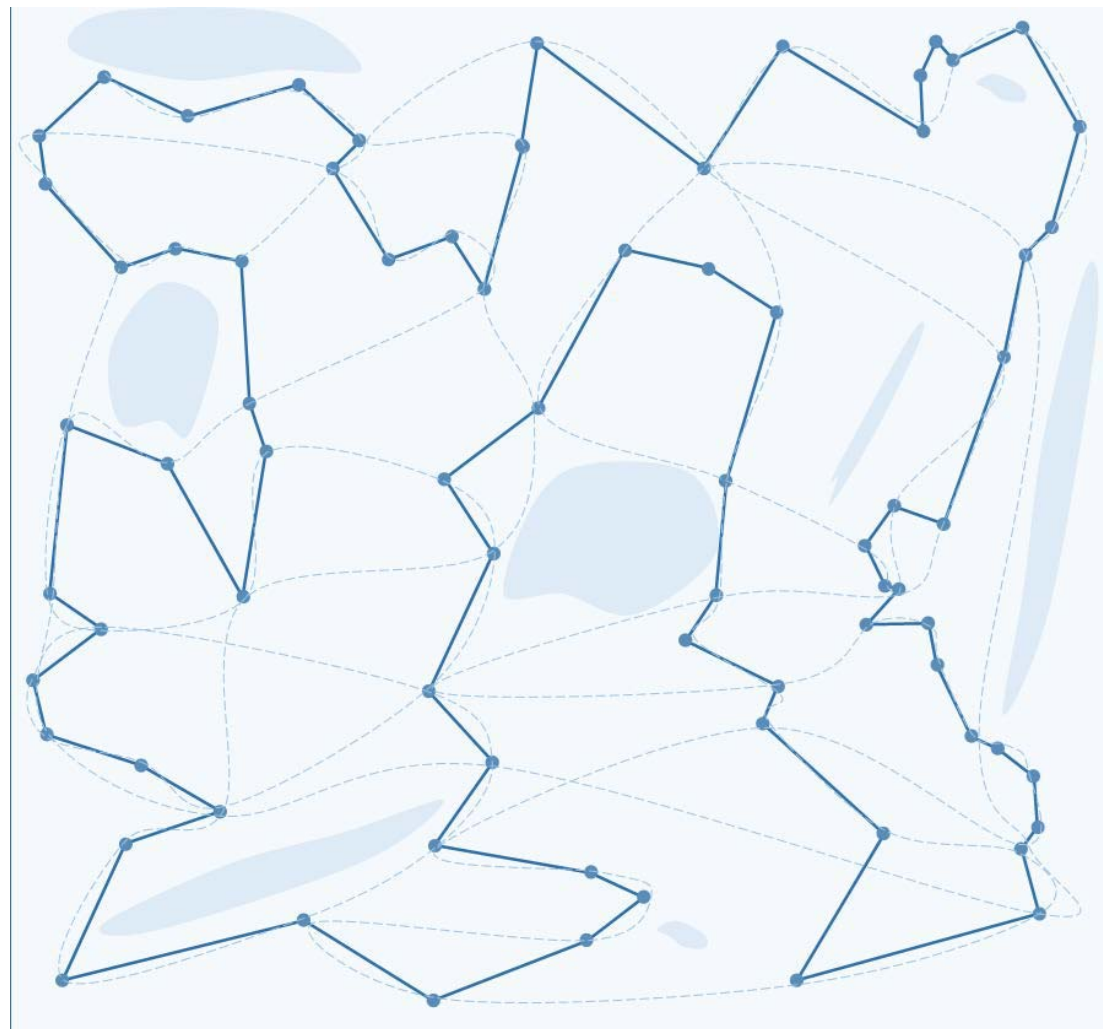

Fig. 3. Optimal transport route for 75 knots.

This example illustrates the use of an optimal method in the logistics. The presented example and figure were made by the authors using a specialized mathematical software package.

\section{Conclusions}

As a result of the study of heuristic methods used in logistics for the construction of optimal routes for freight transportation, the optimal methods are determined. A comparative analysis of ten methods for solving the optimization problem of the NP class traveling salesman showed an estimate of the accuracy and computation time.

The methods of drawing up optimal plans for the delivery of goods allow enterprises to choose convenient delivery routes that meet the specified performance criteria.

The methods imply a quick selection of the optimal delivery routes and distribution of the cargo flow along them. It should be noted the relevance of research in the field of management of logistics processes in the framework of the application of modern information digital technologies. The expansion of logistics enterprises creates great opportunities for improving performance through the use of information technology.

Heuristic methods contribute to the development of management decisions regarding logistics processes.

Digital technologies open up new prospects for the development of the logistics industry, placing emphasis on the increasing demand for and contactless delivery of goods and cargo. 
A significant number of logistics companies consider business digitalization as a way to mitigate emerging risks.

Currently, the automatic control of the logistics process is becoming one of the main trends in the development of rail transport.

Numerical experiments with a simulation model of logistics show the efficiency of the heuristic method.

In the future, the authors plan to develop hybrid methods based on the selected methods to determine the optimal route in digital logistics.

\section{References}

1. I.V. Ilin, D.D. Bolobonov, A.K. Frolov, Proceedings of the 33rd International Business Information Management Association Conference, IBIMA 2019: Education Excellence and Innovation Management through Vision 2020, 5092-5102 (2019)

2. A.I. Levina, A.S. Dubgorn, O.Y. Iliashenko, Proceedings - 2017 European Conference on Electrical Engineering and Computer Science, 351-355 (2018)

3. S. Maydanova, I. Ilin, A. Lepekhin, 33rd International Business Information Management Association Conference, IBIMA 2019: Education Excellence and Innovation Management through Vision 2020, 5103-5111 (2019)

4. P. Baniasadi, M. Foumani, K. Smith-Miles, V. Ejov, European Journal of Operational Research (2020)

5. J. Wu, L. Zhou, Z. Du, Y. Lv, Transportation Research Part E: Logistics and Transportation Review 126, 87-102 (2019)

6. J. Stastný, V. Skorpil, L. Cizek, 39th International Conference on Telecommunications and Signal Processing (2016)

7. S.E. Ivanov, N.N. Gorlushkina, L.N. Ivanova, AIP Conference Proceedings 1952(1), 020043 (2018)

8. T.V. Zudilova, S.E. Ivanov, L.N. Ivanova, International Conference on Current Trends in Computer, Electrical, Electronics and Communication (2017)

9. S.E. Ivanov, N. Gorlushkina, A. Govorov, Procedia Computer Science 136, 210-217 (2018)

10. T. Hoshino, C. Tian, H. Kondo, K. Tsuboi, Y. Hamamatsu, International Journal of Computer Science Issues (IJCSI) 13(3), 1-8 (2016)

11. J. Liu, W. Li, 8th International Conference on Electronics Information and Emergency Communication (ICEIEC) (2018)

12. D.A. Gostevsky, E.V. Konstantinova, Siberian electronic mathematical news 15, 205213 (2018)

13. A. Montero, I. Méndez-Díaz, J.J. Miranda-Bront, Computers \& Operations Research 88, 280-289 (2017)

14. I. Khan, M.K. Maiti, M. Maiti, International conference on mathematics and computing (Springer, Singapore, 2017)

15. P. Medynski, Schriftenreihe des Fachbereichs Informatik der Fachhochschule Dortmund: Band 2, 131 (2016)

16. C. Sun, 9th International Conference on Industrial Technology and Management (ICITM) (2020)

17. Y.C. Hsieh, P.S. You, Appl. Math. Inf. Sci. 6, 371-377 (2012) 
18. A.E.S. Ezugwu, A.O.Adewumi, M.E. Frîncu, Expert Systems with Applications 77, 189210 (2017)

19. O. Mersmann, B. Bischl, J. Bossek, H. Trautmann, M. Wagner, F. Neumann, International Conference on Learning and Intelligent Optimization (Springer, Berlin, Heidelberg, 2012)

20. G.I. Melnikov, N.A. Dudarenko, K.S. Malykh, L.N. Ivanova, V.G. Melnikov, Nonlinear Dynamics and Systems Theory 17(4), 369-375 (2017)

21. D. Kumar, Procedia computer science 132, 824-833 (2018)

22. E. Stripling, S. Vanden Broucke, K. Antonio, B. Baesens, M. Snoeck, Swarm and Evolutionary Computation 40, 116-130 (2018)

23. I. Ilin, S. Maydanova, A. Lepekhin, C. Jahn, J. Weigell, V. Korablev, Lecture Notes in Networks and Systems 157, 179-188 (2021)

24. U. Teschemacher, G. Reinhart, Procedia CIRP 63, 762-767 (2017) 